\title{
Aplicación de sustratos orgánicos en la cría de la lombriz roja californiana (eisenia foetida) para la producción de alimento animal
}

\author{
Application of organic substrates in the breeding of the Californian red \\ worm (eisenia foetida) for the production of animal feed
}

Diana Katherine Campoverde Santos. ${ }^{1}$, Luis Antonio Velasco Matveev. ${ }^{2}$ \& Washington David Acurio Martínez. ${ }^{3}$

Recibido: 21-06-2020 /Aceptado: 20-07-2020 / Publicado: 07-08-2020

\begin{abstract}
.
DOI: https://doi.org/10.33262/concienciadigital.v3i3.1.1354

The objective of the present investigation was to evaluate three different types of substrates. 100 adult Californian red worms Eisenia foetida were placed in all the experimental units. The experimental design was randomized blocks with five replications and three treatments: potato peel, $50 \%$ potato peel and $50 \%$ guinea pig manure, guinea pig manure. The variable earthworm population and weight was analyzed. The substrates were deposited in $0.125 \mathrm{~m}^{3}$ wooden boxes and the composting process was carried out. When obtaining stable temperatures in ranges between 20 to $23{ }^{\circ} \mathrm{C}, 100$ worms were planted per box. After 69 days, enough time for the worms to complete their reproductive cycle, all the worms present in each experimental unit were counted. Significant differences ( $p<0.05 \%$ ) were obtained in the three variables. The substrate "potato peel" registered the highest amount and weight of earthworms (625 earthworms, 937.5g). The mixed substrate $50 \%$ potato peel and $50 \%$ cuy manure recorded the second highest average (189 worms, 264.6g) and thirdly obtained with cuy manure substrate (101 worms, $143.4 \mathrm{~g})$. It was concluded that the potato shell

1 Escuela Superior Politécnica de Chimborazo, Facultad de Ciencias Pecuarias. Riobamba, Ecuador. diana.campoverde@espoch.edu.ec

${ }^{2}$ Escuela Superior Politécnica de Chimborazo, Facultad de Ciencias Pecuarias. Riobamba, Ecuador.

${ }^{3}$ Escuela Superior Politécnica de Chimborazo, Facultad de Ciencias. Riobamba, Ecuador. washington.acurio@espoch.edu.ec
\end{abstract} lvelasco@espoch.edu.ec 
substrate is best suited for worm production, subsequently with information obtained from the number of worms classified by stage (cocon, juvenile, sub-adult and adult), a mathematical model defined by Peña (2004) was used for the purpose of estimating the probabilistic population dynamics of survival over the next 20 weeks.

Keywords: Potato shell, cuy manure, Eisenia foetide, mathematical model, population dynamics

\section{Resumen.}

El objetivo de la presente investigación fue evaluar tres tipos diferentes de sustratos, en todas las unidades experimentales se colocaron 100 lombrices rojas californianas adultas Eisenia foetida. El diseño experimental fue de bloques al azar con cinco repeticiones y tres tratamientos: cáscara de papa, 50\% de cáscara de papa y $50 \%$ de estiércol de cuy. Se analizó la variable población total y peso de lombrices. Los sustratos fueron depositados en cajas de madera de $0.125 \mathrm{~m}^{3}$ y se realizó el proceso de compostaje. Al obtener temperaturas estables en rangos de entre 20 a $23{ }^{\circ} \mathrm{C}$ se realizó la siembra de 100 lombrices por caja. Transcurridos 70 días, tiempo suficiente para que las lombrices cumplan su ciclo reproductivo se procedió a contar todas las lombrices presentes en cada unidad experimental. Se obtuvieron diferencias significativas $(\mathrm{p}<0.05 \%)$ en las tres variables. El sustrato "cáscara de papa" registró en promedio la mayor cantidad y peso de lombrices (625 lombrices, $937.5 \mathrm{~g}$ ). El sustrato mixto $50 \%$ de cáscara de papa y 50\% de estiércol de cuy registró el segundo mejor promedio (189 lombrices, 264.6g) y en tercer lugar se obtuvo con el sustrato estiércol de cuy (101 lombrices, $143.4 \mathrm{~g}$ ). Se concluyó que el sustrato cáscara de papa es el más adecuado para la producción de lombriz, posteriormente con la información obtenida del número de lombrices clasificados por estadío (cocon, juvenil, sub adulto y adulto), se utilizó un modelo matemático definido por Peña (2004) con el propósito de estimar la dinámica poblacional probabilística de supervivencia de las próximas 20 semanas.

Palabras claves: Cáscara de papa, estiércol de cuy, Eisenia foetida, modelo matemático, dinámica poblacional

\section{Introducción.}

En la actualidad de acuerdo con varios autores se conocen aproximadamente 8.000 variedades de lombrices, pero sólo 3.500 de ellas han sido estudiadas y clasificadas. De estas 3.500 variedades, unas pocas han sido domesticadas y adaptadas en criaderos, para consumar la función que en forma natural efectúan en la tierra como es la digestión de la materia orgánica y los microorganismos, trabajando en forma intensiva y generando un valioso producto, que es el humus de lombriz, además que son apreciadas por su alto contenido de 
proteínas (Agroflor, s.f; Chávez y Fuentes, 2013; Ayra y Domínguez, 2010; Peñaranda, 2006). En países como Europa, Japón y Estados Unidos se han desarrollado criaderos de lombrices, siendo la especie más utilizada y la que mejor resultados ha presentado la especie Eisenia Foétida.

El manejo de los procesos naturales relacionados con la lombricultora que es la técnica orgánica que permite favorecer su dinámica para obtener un impacto efectivo en el ámbito social, agrícola y económico (Hatti et al., 2010; Gheisari et al., 2010), dicha técnica permite crear alternativas sostenibles de la aplicación del humus de lombriz como abono en diferentes cultivos. (Sarmiento, 2019)

La explotación de los sustratos o mezclas ricas en fibras cortejados de una adecuada tecnología de cultivo se puede lograr una alta tasa de crecimiento y reproducción, por ende, generar una mejor producción altamente representativa de la biomasa de lombriz, generando fuentes de proteína no convencionales sin que afecte directamente a la calidad del producto que podría ser humus o biofertilizante (García et al. 1996, Chávez y Fuentes, 2013; Aira y Domínguez, 2010; Orozco et al, 1996).

Es por su importancia ecológica que a los estudiantes de las escuelas, colegios y universidad se les enseña el manejo de la lombriz como una alternativa para cuidar el medio ambiente, coadyudando al planteamiento de proyectos de emprendimiento, ante esto Peña (2004) realiza un estudio sobre el comportamiento poblacional lombrícola, considerando las diferentes circunstancias ecológicas, y mediante una simulación establece un modelo para estimar una dinámica poblacional a partir de datos empíricos obtenidos de las condiciones ideales de crianza, logrando así una ecuación que permite orientar a quienes elijan implementar la lombricultora en los futuros sistemas de producción.

De igual manera existe actualmente una corriente de los consumidores en adquirir productos de origen animal que sean alimentados de forma natural y orgánica, misma que necesita de materias primas que provean nutrientes adecuados para lograr rendimientos óptimos.

La producción tradicional de aves de traspatio es una actividad de subsistencia que utiliza pavos criollos y gallinas. La suplementación de las aves de traspatio con lombrices es la alternativa viable para la producción de huevo y carne, que además de mejorar la alimentación de la familia, se generan ingresos adicionales por la venta de huevo, pollos y biofertilizante. (Vargas et al., s.f.).

El análisis bromatológico de la lombriz roja reporta un contenido de humedad aproximado del 88,07\%, y un contenido de nutrientes en base seca de: Proteínas 58.87\%, Cenizas 13,53\%, Grasas $7.94 \%$, Carbohidratos $19.63 \%$. Su proteína es de alto valor biológico ya que está compuesta por aminoácidos esenciales como la Valina 5\%, Metionina 1,6\%, Lisina 6,6\%, Arginina 6,1\%. En el Ecuador las provincias de Azuay, Guayas, Los Ríos, Pichincha y 
Tungurahua son las que llevan adelante la práctica de la lombricultora, se encuentran en el oeste y centro del país, se caracterizan por tener un clima favorable para la producción de humus. La agricultura es una de las principales fuentes de sustento de los habitantes mochanos, posee tierra fértil ideal para el cultivo de papas, cebolla colorada, maíz, habas, entre otros, y entre sus platos típicos se encuentran el cuy asado junto con papas con cuero.

En el cantón Mocha las cáscaras de las papas y el estiércol del cuy son arrojados a la basura o abandonados en los campos para ser degradados, lo cual puede ser una fuente de contaminación importante del suelo y el agua además que atrae la presencia de plagas. Por lo que la lumbricultura se ve como una opción viable de manejo de estos desperdicios y una alternativa a los procesos de compostaje con fase termofílica, los cuales producen cantidades significativas de gases de efecto invernadero (Romero, Ocampo, Sandoval, \& Tobar, 2018).

La presente investigación tuvo como objetivo evaluar el uso de diferentes sustratos procedentes de residuos sólidos orgánicos en la producción de la lombriz roja californiana Eisenia foetida para la producción de alimento animal, una vez que se selecciona el mejor sustrato que maximice la producción de lombriz, se utilizó el modelo de estimación establecido por Ferruzi (2001) de la dinámica de la población para estimar la tasa de crecimiento exponencial para los próximos 140 días (20 semanas) a partir de los datos obtenidos experimentalmente en los primeros 70 días.

\section{Metodología.}

El estudio tuvo lugar en el cantón Mocha ubicado al sureste de la ciudad de Ambato, Provincia de Tungurahua, con una superficie de $82,3 \mathrm{~km}^{2}$, repartidos en la parroquia central Mocha con $76 \mathrm{Km} 2$ y 6,3 km2 en Pinguilí. Se realizó a dos cuadras del Parque Cívico del Cantón en la Avenida 13 de mayo y el Rey. Se utilizó un diseño completamente al azar con cinco repeticiones y tres tratamientos: cáscara de papa, 50\% de cáscara de papa y 50\% de estiércol de cuy. El diseño aplicado cumplió con los supuestos de homogeneidad de varianzas, aleatoriedad e independencia de los errores, posteriormente identificando el mejor tratamiento con los datos observados se procedió a estimar la dinámica poblacional probabilística

\section{Pre compostaje}

El trabajo de estudio se realizó en un área de $0.5 \mathrm{~m}$ largo x $0.5 \mathrm{~m}$ de ancho x $0.5 \mathrm{~m}$ de profundidad $=0.125 \mathrm{~m}^{3}$, el terreno del área de estudio fue dividido en 15 parcelas, cada parcela representa la unidad experimental. Se distribuyeron 100 lombrices en cada parcela, mediante un diseño completamente al azar en tres tratamientos, con cinco repeticiones, los tratamientos considerados en el estudio son: sustrato A (cáscara de papas -4 $500 \mathrm{~g}$ ), sustrato 
B (una porción mixta de cáscara de papas -2 $250 \mathrm{~g}+$ estiércol de cuy -2 250g) y sustrato C (estiércol de cuy- 4500 g), considerando temperaturas estables en rangos de entre 20 a 23 ${ }^{\circ} \mathrm{C}$. Transcurridas 10 semanas, tiempo suficiente para que las lombrices cumplan su ciclo reproductivo se procedió a contar todas las lombrices presentes en cada unidad experimental, considerando el número de lombrices por estadío (cocon, juvenil, sub adulto y adulto). De tal manera que estos registros permitan estimar la dinámica poblacional de las próximas 20 semanas usando el modelo matemático probabilístico de supervivencia formulado por Peña (2004).

\section{Análisis estadístico}

Los datos obtenidos fueron analizados estadísticamente, para lo cual se aplicó un análisis de varianzas procesados mediante el software libre RStudio, a la vez se realizó: pruebas de hipótesis y de comparación múltiple con un nivel de significancia de 0.05.

\section{Modelo Matemático}

En un estudio realizado por Peña (2004), para estimar la dinámica de una población, presenta un modelo de 13 variables de categorías, basado en el cálculo de probabilidades presentado por Johnson (1983), las cuales se resumen de manera general en las 2 ecuaciones descritas con las siguientes ecuaciones diferenciales:

$$
\begin{gathered}
\frac{d L_{1_{n-1}}}{d t}=\beta_{x}-\delta_{x_{1}} \cdot L_{1_{n-1}}-l_{x_{1}+1} \cdot L_{1_{n-1}} \\
\frac{d L_{2 n-1}}{d t}=l_{x_{1}+1} \cdot L_{1_{n-1}}-\delta_{x_{2}} \cdot L_{2_{n-1}}-l_{x_{2}+1} \cdot L_{2_{n-1}}
\end{gathered}
$$

Donde: $\beta_{x}=\mu_{x} \cdot l_{x_{n}+1}, l$

$\beta_{x}=$ es la natalidad en unidades de cocones/semanas

$\mu_{x}=1$ en unidades cocones/lombrices

$l_{x_{n}+1}=$ representa a los sobrevivientes de cada una de las categorías con " $\mathrm{n}$ " orden.

$P_{x_{n}}=\frac{l_{x_{n}+1}}{l_{x_{n}}}$

$l_{x_{n}-1}=$ es el número de individuos o población que se encuentra en cada categoría, para cada estadío (cocon, juvenil, subadulto y adulto) como 


\section{Resultados}

Con el propósito de identificar cual es el sustrato más adecuado en base a los desperdicios comunes que se encuentran en la zona, para la producción de lombrices se realizó el estudio de 15 parcelas con tres sustratos distintos, evidenciando así los siguientes resultados.

Los tipos de sustratos indicaron su efecto significativo, con diferencias $(\mathrm{p}<0.05)$ en el número de lombrices (Tabla 1) entre tratamientos.

Al aplicar la prueba de separación de medias Duncan a los tratamientos (0.05 significancia) indica que el sustrato A (cáscara de papas - $4500 \mathrm{~g}$ ) estadísticamente es el mejor sustrato para la reproducción de lombrices produciendo 625 lombrices en $0.125 \mathrm{~m}^{3}$, seguido del sustrato B (una porción mixta de cáscara de papas - $2250 \mathrm{~g}+$ estiércol de cuy - $2250 \mathrm{~g}$ ) la que produjo 189 lombrices $0.125 \mathrm{~m}^{3}$, y por último tenemos al sustrato C (estiércol de cuy - $4.500 \mathrm{~g}$ ) que produjo 101 lombrices en $0.125 \mathrm{~m}^{3}$.

Tabla 1. Análisis de Varianza del Número de Lombrices

\begin{tabular}{lccccc}
\hline \multicolumn{1}{c}{ FV } & SC & GL & CM & Fo & Valor $\mathbf{p}$ \\
\hline Tratamiento (Sustrato) & 787360 & 2 & 393680 & 884.67 & $<0,000$ \\
Error & 5340 & 12 & 445 & & \\
Total & 792700 & 14 & & & \\
\hline
\end{tabular}

Fuente: Elaboración Propia.

Tabla 2. Cantidad promedio de lombrices por sustrato utilizado

\begin{tabular}{cc}
\hline Sustrato & Número de lombrices \\
\hline Cáscara de Papa & 625 \\
$50 \%$ de cáscara de papa y & 189 \\
$50 \%$ de estiércol de cuy & \\
Estiércol de cuy & 101 \\
\hline
\end{tabular}

Fuente: Elaboración Propia. 


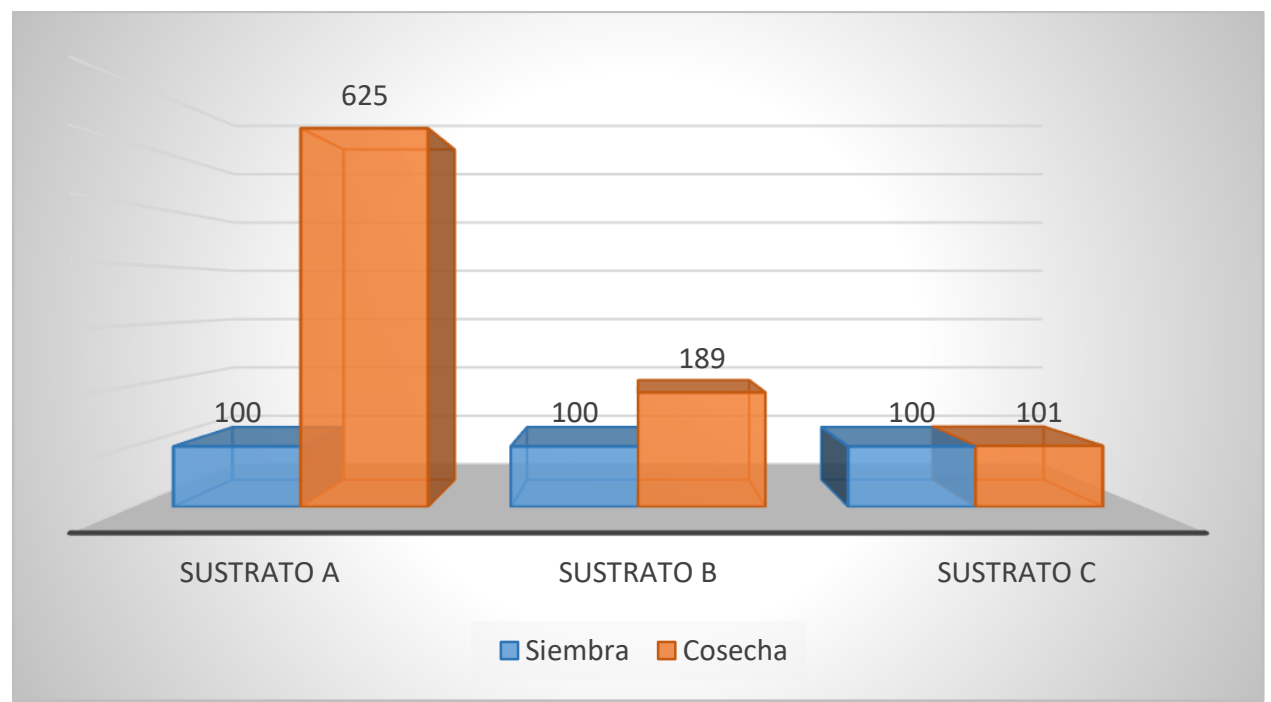

Figura 1. Cantidad promedio de lombrices por sustrato utilizado Fuente: Elaboración Propia.

El resultado obtenido concuerda con lo expresado por Carrillo et al, (2017) respecto al mejor rendimiento obtenido con el sustrato cáscara de papa ya que las altas concentraciones de nitrógeno y bajas concentraciones de carbono que posee el estiércol de los animales herbívoros limitan la reproducción de las lombrices.

\section{Peso de lombrices}

Respecto al peso total de las lombrices el sustrato A (cáscara de papas - $4500 \mathrm{~g}$ ) estadísticamente es el mejor sustrato para la producción de lombrices produciendo $937.5 \mathrm{~g}$ de lombrices en $0.125 \mathrm{~m}^{3}$, seguido del sustrato B (una porción mixta de cáscara de papas-2 $250 \mathrm{~g}$ + estiércol de cuy - $2250 \mathrm{~g}$ ) la que produjo $264.6 \mathrm{~g}$ de lombrices $0.125 \mathrm{~m}^{3}$, y por último tenemos al sustrato $\mathrm{C}$ (estiércol de cuy-4 $500 \mathrm{~g}$ ) que produjo $143.4 \mathrm{~g}$ de lombrices en 0.125 $\mathrm{m}^{3}$.

Tabla 3. Peso promedio de lombrices por sustrato utilizado

\begin{tabular}{cc}
\hline Sustrato & $\begin{array}{c}\text { Peso en } \\
\text { gramos }\end{array}$ \\
\hline Cáscara de Papa & 937.5 \\
$50 \%$ de cáscara de papa y & 264.6 \\
$50 \%$ de estiércol de cuy & \\
Estiércol de cuy & 143.4 \\
& \\
\hline
\end{tabular}

Fuente: Elaboración Propia. 


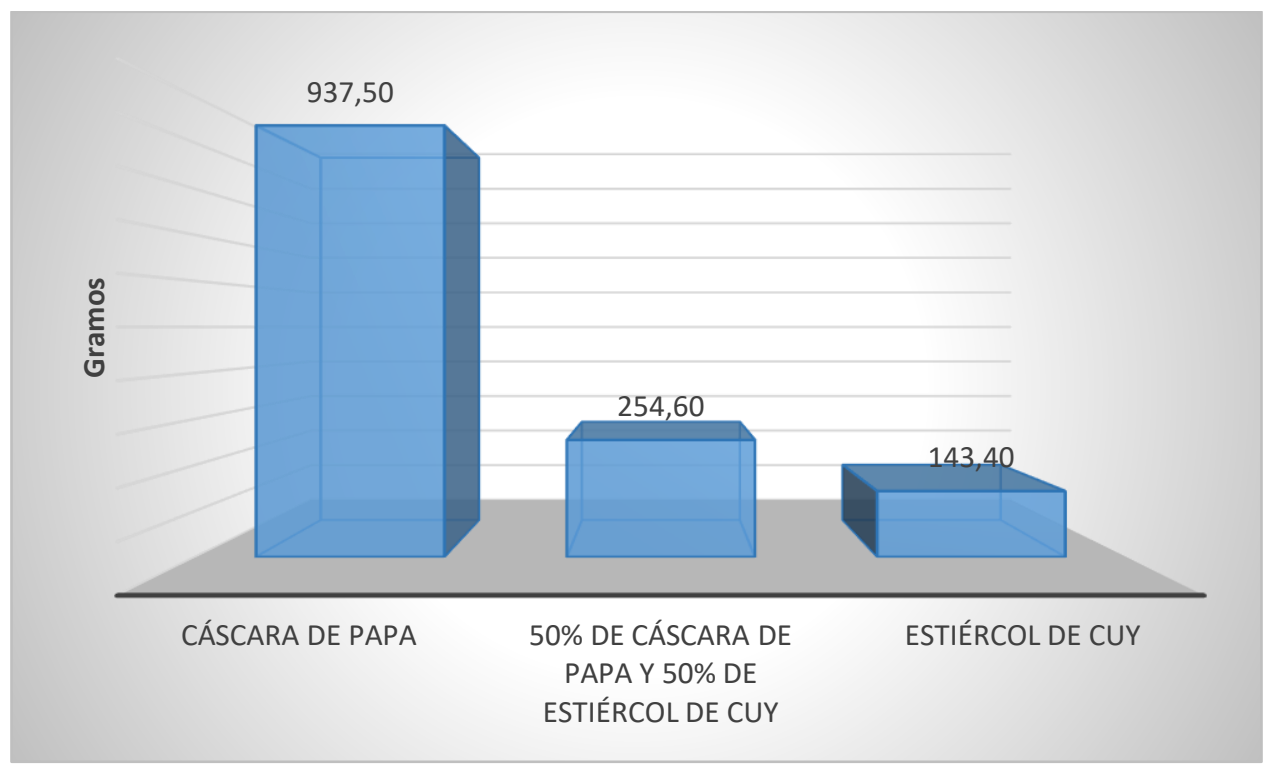

Figura 2. Peso de lombrices por sustrato utilizado

Fuente: Elaboración Propia.

El resultado obtenido concuerda con lo expresado por Carrillo et al (2017) respecto al mejor rendimiento obtenido con el sustrato cáscara de papa, esto puede deberse como consecuencia de la dieta de los animales, sus excretas pueden contener elevadas concentraciones de gases tóxicos como Dióxido de Carbono, Metano y Óxido Nitroso, que modifican los nutrientes disponibles para las lombrices, lo cual se torna perjudicial e impide el correcto desarrollo de estas.

Además, concuerda con las investigaciones realizadas por varios autores por en el que relaciona directamente a la presencia de proteínas resistentes a determinados ataques enzimáticos en los sustratos elaborados con excretas animales y las condiciones inadecuadas de temperatura; además análogamente establecen la relación adecuada entre las lombrices rojas californianas con la glucosa presente en los sustratos de origen vegetal (Morales, et al., 2009; Piza, 2017; Rivera y Yate, 2019; Cárdenas, 2009; Cajas, 2009).

\section{Aporte nutricional de la lombriz}

En base al análisis bromatológico de la Lombriz Roja Californiana (Eisenia Foetida) realizado por la Facultad de Biología de la Universidad Santiago de Compostela, se realizó una conversión del peso de las lombrices obtenido en la presente investigación, dando como resultado que el Sustrato A (cáscara de papa) produjo $65.84 \mathrm{~g}$ de Proteína, $15.15 \mathrm{~g}$ de Cenizas, $8.38 \mathrm{~g}$ de grasa y $21.95 \mathrm{~g}$ de Carbohidratos en $0.125 \mathrm{~m}^{3}$. 
El sustrato B ( $50 \%$ de cáscara de papa y $50 \%$ de estiércol de cuy) produjo $18.6 \mathrm{~g}$ de proteína, $4.28 \mathrm{~g}$ de Cenizas, $2.51 \mathrm{~g}$ de Grasa y $6.2 \mathrm{~g}$ de Carbohidratos en $0.125 \mathrm{~m}^{3}$.

El sustrato C (estiércol de cuy) produjo 10.07 g de Proteína, $1.25 \mathrm{~g}$ de Cenizas, $1.25 \mathrm{~g}$ de Grasa y $3.35 \mathrm{~g}$ de Carbohidratos en $0.125 \mathrm{~m}^{3}$.

Tabla 4. Aporte nutricional de la lombriz

\begin{tabular}{cccccc}
\hline Sustrato & $\begin{array}{c}\text { Peso } \\
\text { fresco }\end{array}$ & $\begin{array}{c}\text { \%Proteínas } \\
\text { en base } \\
\text { seca }\end{array}$ & $\begin{array}{c}\text { \% Cenizas } \\
\text { en base } \\
\text { seca }\end{array}$ & $\begin{array}{c}\text { \% Grasas en } \\
\text { base seca }\end{array}$ & $\begin{array}{c}\% \\
\text { Carbohidratos } \\
\text { en base seca }\end{array}$ \\
\hline Cáscara de Papa & $937.5 \mathrm{~g}$ & $65.84 \mathrm{~g}$ & $15.13 \mathrm{~g}$ & $8.38 \mathrm{~g}$ & $21.95 \mathrm{~g}$ \\
$\begin{array}{c}50 \% \text { de cáscara de } \\
\text { papa y } 50 \% \text { de } \\
\text { estiércol de cuy }\end{array}$ & $264.6 \mathrm{~g}$ & $18.60 \mathrm{~g}$ & $4.28 \mathrm{~g}$ & $2.51 \mathrm{~g}$ & $6.20 \mathrm{~g}$ \\
$\begin{array}{c}\text { Estiércol de cuy } \\
\text { s }\end{array}$ & $143.4 \mathrm{~g}$ & $10.07 \mathrm{~g}$ & $2.31 \mathrm{~g}$ & $1.25 \mathrm{~g}$ & $3.35 \mathrm{~g}$ \\
\hline
\end{tabular}

Fuente: Elaboración Propia.

El alto contenido proteico, proporciona la alternativa de alimentación de aves, peces y otros animales domésticos, además se sintetiza a partir de desechos orgánicos siendo una proteína de bajo costo. (Guerra, 2011; Isea et al., 2008, Gonzalvo et al., 2001, Salinas et al. 2014)

Posterior al proceso de determinación del sustrato adecuado, se estimaron los parámetros de natalidad, mortalidad y probabilidad de supervivencia por cada estadío (categorías de edades de las lombrices), considerando la población inicial y la tasa de eclosión durante las 10 semanas, se utilizó el modelo establecido por Peña (2008) para estimar la dinámica poblacional usando probabilidades de supervivencia con los datos obtenidos en el experimento en las próximas 20 semanas (Figura 3), en un principio de 0 a 10 semana se presenta un crecimiento lento para todos los estadíos, a partir de las 20 semanas ya se empieza a notar un crecimiento exponencial mayor de cada tipo de estadío.

Al iniciar la etapa de eclosión aumenta la densidad poblacional, y de esta manera aumenta también la demanda de alimento y espacio, razón por la cual las lombrices adultas se dispersan y esto causa mayor dificultad para el encuentro y apareamiento entre dos animales adultos sexualmente activos, ocasionando temporalmente un descenso poblacional. Cuando la progenie llega a una edad adulta y madurez reproductiva, se equilibra el conteo poblacional y comienza a evolucionar adecuadamente el proceso poblacional y reproductivo. 


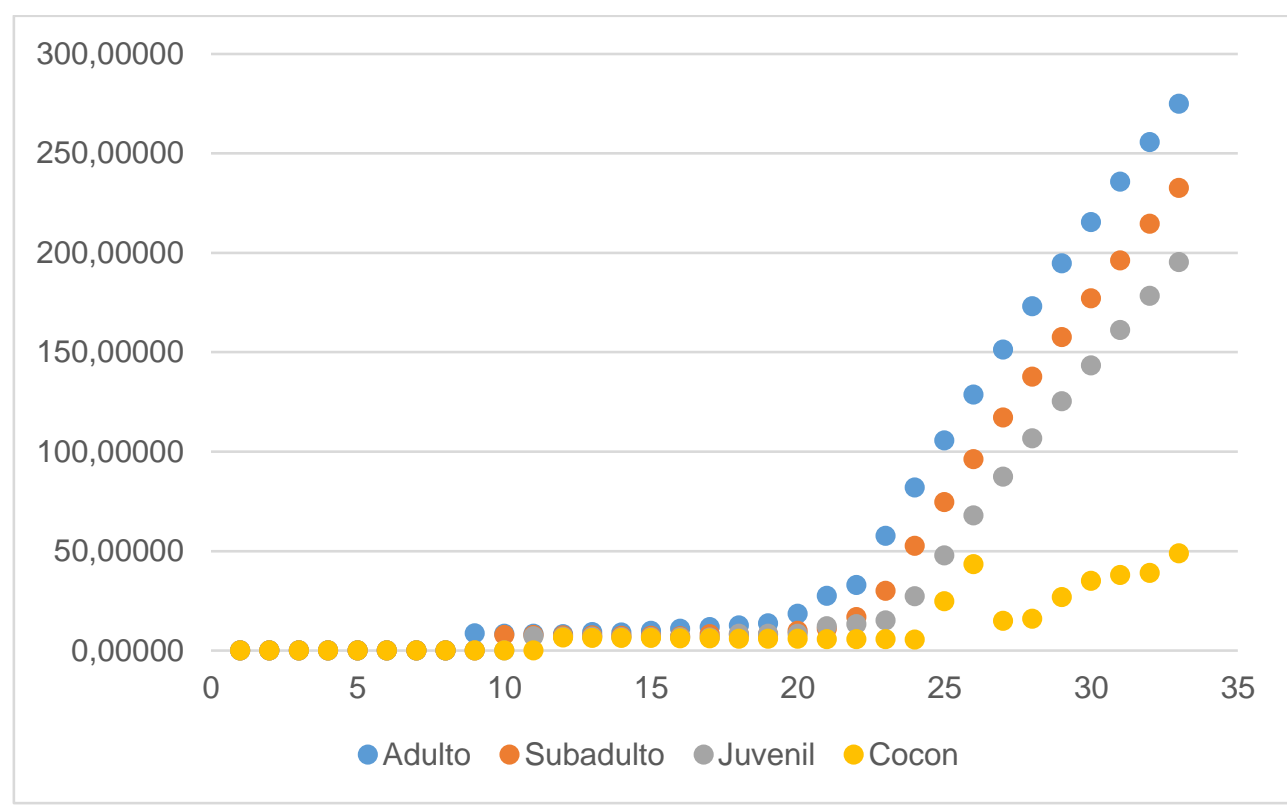

Figura 3. Crecimiento exponencial de lombrices por categoría (Probabilidad de supervivencia)

Fuente: Elaboración Propia.

\section{Conclusiones}

- El comportamiento productivo y reproductivo de la lombriz roja californiana Eisenia foetida está directamente relacionado con el tipo de sustrato utilizado en la presente investigación.

- Las técnicas del diseño experimental permitieron diagnosticar que dentro del lapso de 10 semanas el tratamiento que permite una mayor producción de lombrices (biomasa) en el menor tiempo posible es el sustrato Cáscara de papas, ya que a partir de 100 lombrices se obtuvo un promedio de 625 lombrices con un peso de $937.5 \mathrm{~g}$ y un aporte de proteínas de $65,84 \mathrm{~g}$, todo esto producido en un volumen de terreno de $0,125 \mathrm{~m}^{3}$.

- La lombricultura es una alternativa interesante para la producción de alimento natural para animales de traspatio o de crianza orgánica ya que provee de proteínas de alto valor biológico, requiere de poco espacio, utiliza desechos comunes y además puede ser generador de otras fuentes de ingresos económicos por la venta de humus, lombrices, etc.

- El proceso de producción de lombriz roja californiana es un proceso sencillo y que puede ser implementado por todas aquellas personas que deseen contribuir a la disminución de la contaminación ambiental procurando un manejo adecuado de los residuos orgánicos, permite ahorrar debido a que no es necesario comprar fertilizantes artificiales.

- Además, con el modelo de estimación, se puede establecer cuál es la dinámica de la 
población probabilística ideal de la lombriz roja, permitiéndonos conocer características propias del aumento poblacional por cada tipo de estadío (cocon, juvenil, subadulto y adulto) de Eisenia Foetida y por supuesto determinar los cambios en la población con el paso del tiempo.

\section{Referencias bibliográficas.}

Agroflor (s.f). Manual lombricultura. Recuperado de http://agro.unc.edu.ar/ biblio/Manual\%20de\%20Lombricultura.pdf

Cajas, S. (2009). Efecto de la utilización de aserrín en combinación con bovino, como sustrato en la producción de humus de lombriz Eisenia Foetida. Chimborazo Riobamba: Escuela Superior Politécnica de Chimborazo, Tesis de grado para la obtención del título de Ingeniero Zootecnista.

Cardenas, B. E. (2014). Guía técnica para el aprovechamiento de residuos orgánicos, a través de metodologías de compostaje y lombricultura. Colombia: Universidad Nacional de Colombia y Alcaldía Mayor de Bogotá.

Chávez, C., \& Fuentes, A. (2013). Determinación de parámetros fisicoquímicos y microbiológicos del lixiviado obtenidos del estiércol de bovino utilizando Eisenia Foetida (lombriz roja californiana). San Salvador-El Salvador: Universidad de El Salvador.

Ferruzzi C. (2001). Manual de Lombricultura. Tercera Reimpresiön. Ediciones Mundi prensa. Barcelona, España.

Gheisari, S., Danesh, S., \& Mousavi, S. M. (2010). Growth and reproduction of Eisenia fetida in vermicomposting of organic fraction of municipal solid wastes. Asian Journal of Chemistry, 22(2), 1266-1274.

Guerra Aznay, M. (2011). Humus de lombriz Eisenia foetida para cultivar dos microalgas marinas como alimento de larvas de camarón (Master's thesis). La Habana: Universidad de la Habana-Centro de investigaciones marinas.

Gheisari, S.; Danesh, S.; Mousavi, S. (2010). Growth and Reproduction of Eisenia foetida in Vermicomposting of Organic Fraction of Municipal Solid Wastes. Asian Journal of Chemistry, 22 (2), 1266-1274.

Gonzalvo, S., Nieves, D., Ly, J., Macías, M., Carón, M., \& Martínez, V. (2001). Algunos aspectos del valor nutritivo de alimentos venezolanos destinados a animales monogástricos. Livestock Research for Rural Development, 13(2), 25.

Hatti, S., Londonkar, R., Patil, S., Gangawane, A., \& Patil, C. (2010). Effect of Eisenia fetida vermiwash on the growth of plants. Journal of crop Science, 1(1), 6. 
Isea, F., Blé, C., Medina, A. L., Aguirre, P., Bianchi, G., \& Kaushik, S. (2008). Estudio de digestibilidad aparente de la harina de lombriz (Eisenia andrei) en la alimentación de trucha arco iris (Onchorinchus mykiss). Revista chilena de nutrición, 35(1), 62-68.

Johnson, I., \& Thornley, J. (1983). Vegetative crop growth model incorporating leaf area expansion and senescence, and applied to grass. Plant, Cell \& Environment, 6(9), 721-729.

Morales, J., Fernández, M., Montiel, A., \& Peralta, B. C. (2009). Evaluación de sustratos orgánicos en la producción de lombricomposta y el desarrollo de lombriz (Eisenia foetida). Biotecnia, 11(1), 19-26.

Orozco, F. H., Cegarra, J., Trujillo, L. M., \& Roig, A. (1996). Vermicomposting of coffee pulp using the earthworm Eisenia fetida: effects on $\mathrm{C}$ and $\mathrm{N}$ contents and the availability of nutrients. Biology and fertility of soils, 22(1-2), 162-166.

Peña, F. (2004) Un modelo de simulación para estimar la dinámica de una población ideal de Eisenia foetida (Lombriz de tierra roja californiana). México: Universidad Autónoma Agraria "Antonio Narro".

Peñaranda, G. (2006) Técnicas y aplicaciones del cultivo de la lombriz roja californiana (Eisenia Foetida). Curso Teórico y Práctico de Lombricultura. Academia de Ciencias de Ucrania, Kiev, Ucrania. Edit. Revista Procampo-N27.Lima, Perú.

Piza Castillo, C. R. (2017). Determinación de la calidad de humus de lombriz roja californiana (Eisenia foetida) a partir de dos procesos en el tratamiento de alimento ofertado (Doctoral dissertation). Bolibvia-La Paz: La Universidad Mayor de San Andrés.

Rivera, P.0, \& Yate, A. (2019). Uso de eisenia foetida (oligoquetos: lumbricidae) para la producción de bioabono, Bogotá-Colombia. RIAA, 10(2), 1.

Romero, C., Ocampo, J., Sandoval, E., \& Tobar, J. (2018). Evaluación de sustratos para la producción de lombriz de tierra (Eisenia foetida). Centro Agrícola, 45(4), 68-74.

Sarmiento, G., Pino, D., Mena, L., Medina, H., Lipa, L. (2019). Aplicación de humus de lombriz y algas marinas en el cultivo de sandía (Citrullus lanatus Thunb.) var. Santa Amelia. Scientia Agropecuaria, 10(3), 363 - 368.

Salinas, F., Sepúlveda, L., \& Sepúlveda-Chavera, G. (2014). Evaluación de la calidad química del humus de lombriz roja californiana (Eiseniafoetida) elaborado a partir de cuatro sustratos orgánicos en Arica. Idesia (Arica), 32(2), 95-99.

Shields, B. E. (1999). Raising Earthworms for Profit. Twentieth Edition. Shields Publications. USA $128 \mathrm{P}$. 
Vargas López, S, García Martínez, A., Palma, H., Librado, M., (s.f.). Integración de la lombricultura en la producción de aves de aves de traspatio en Puebla, México: Universitat De Les Illes Balears, Recuperado de fci.uib.es/Servicios/libros/conferencias/seae/Integracion-de-la-lombricultura-en-laproduccion.cid221661. 


\section{PARA CITAR EL ARTÍCULO INDEXADO.}

Diana Katherine , C. S., Luis Antonio , V. M., \& Washington David, A. M. (2020). Aplicación de sustratos orgánicos en la cría de la lombriz roja californiana (eisenia foetida) para la producción de $\begin{array}{llll}\text { alimento animal } \quad \text { ConcienciaDigital, } & 3(3.1),\end{array}$ https://doi.org/10.33262/concienciadigital.v3i3.1.1354

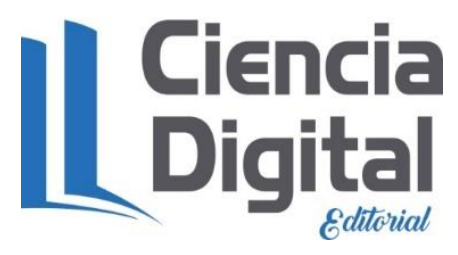

El artículo que se publica es de exclusiva responsabilidad de los autores y no necesariamente reflejan el pensamiento de la Revista Conciencia Digital.

El artículo queda en propiedad de la revista y, por tanto, su publicación parcial y/o total en otro medio tiene que ser autorizado por el director de la Revista Conciencia Digital.

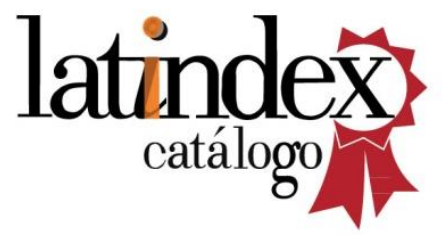

\section{Conciencia}

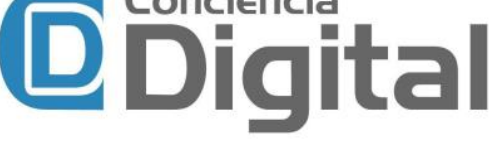

